Klaus von Heusinger, Claudia Maienborn and Paul Portner (Eds.)

Semantics - Typology, Diachrony and Processing 
This volume is part of a larger set of handbooks to Semantics

1 Semantics: Foundations, History and Methods

Klaus von Heusinger, Claudia Maienborn, Paul Portner (eds.)

2 Semantics: Lexical Structures and Adjectives

Claudia Maienborn, Klaus von Heusinger, Paul Portner (eds.)

3 Semantics: Theories

Claudia Maienborn, Klaus von Heusinger, Paul Portner (eds.)

$4 \quad$ Semantics: Noun Phrases and Verb Phrases

Paul Portner, Klaus von Heusinger, Claudia Maienborn (eds.)

5 Semantics: Sentence and Information Structure

Paul Portner, Claudia Maienborn, Klaus von Heusinger (eds.)

6 Semantics: Interfaces

Claudia Maienborn, Klaus von Heusinger, Paul Portner (eds.)

7 Semantics: Typology, Diachrony and Processing

Klaus von Heusinger, Claudia Maienborn, Paul Portner (eds.) 


\section{Semantics \\ Typology, Diachrony \\ and Processing}

Edited by

Klaus von Heusinger

Claudia Maienborn

Paul Portner

DE GRUYTER

MOUTON 
ISBN 978-3-11-058724-1

e-ISBN (PDF) 978-3-11-058982-5

e-ISBN (EPUB) 978-3-11-058732-6

\section{Library of Congress Cataloging-in-Publication Data}

Names: Heusinger, Klaus von, editor. | Maienborn, Claudia, editor. | Portner, Paul, editor.

Title: Semantics : typology, diachrony and processing / edited by Klaus von Heusinger, Claudia Maienborn, Paul Portner.

Description: Berlin ; Boston : De Gruyter, [2019] | Series: Mouton reader | Includes bibliographical references and index.

Identifiers: LCCN 2018031263 (print) | LCCN 2018047130 (ebook) | ISBN

9783110589825 (electronic Portable Document Format (pdf) | ISBN

9783110587241 (paperback) | ISBN 9783110589825 (e-book pdf) |ISBN

9783110587326 (e-book epub)

Subjects: LCSH: Semantics. | BISAC: LANGUAGE ARTS \& DISCIPLINES / Linguistics / Semantics.

Classification: LCC P325 (ebook) | LCC P325 .S37999 2019 (print) | DDC 401/.43--dc23

LC record available at https://lccn.loc.gov/2018031263

\section{Bibliographic information published by the Deutsche Nationalbibliothek}

The Deutsche Nationalbibliothek lists this publication in the Deutsche Nationalbibliografie; detailed bibliographic data are available in the Internet at http://dnb.dnb.de.

(C) 2019 Walter de Gruyter GmbH, Berlin/Boston

Cover image: skyNext/iStock / Getty Images Plus Typesetting: Integra Software Services Pvt. Ltd. Printing and Binding: $\mathrm{CPI}$ books $\mathrm{GmbH}$, Leck

www.degruyter.com 